\title{
A critical comparison of model-predictive and pseudospectral control for wave energy devices
}

\author{
Romain Genest $^{1} \cdot$ John V. Ringwood ${ }^{1}$
}

Received: 29 February 2016 / Accepted: 27 May 2016 / Published online: 14 June 2016

(C) Springer International Publishing Switzerland 2016

\begin{abstract}
Model predictive control (MPC) and pseudospectral optimal control (PSC) have been proposed over the past decade to maximise energy capture for wave energy devices. Both philosophies share a similar cost function and both can deal with constraints on system (displacement, velocity) and control (force) variables. Recently, a receding horizon version of the PSC method (RHPSC) has been developed, permitting a direct comparison between MPC and RHPSC formulations. This paper demonstrates that while the control objectives are very similar, the numerical properties of both algorithms are quite different, having implications for practical use, both in terms of performance and implementation issues.
\end{abstract}

Keywords Wave energy control · Model predictive control · Pseudospectral optimal control · Comparison

\section{Introduction}

The emergence of MPC in the process industries, particularly in oil and petrochemical plants, occurred in the 80s; the implemented algorithms were initially based on optimal control methodologies developed by Richalet et al. (1978) and Cutler and Ramaker (1980). Such applications, described by slow dynamic models were, at that time, particularly well suited for MPC algorithms. MPC offers an interesting alternative to solve multivariable optimal control problems

Romain Genest

rgenest@eeng.nuim.ie

John V. Ringwood

john.ringwood@eeng.nuim.ie

1 Centre for Ocean Energy Research, Maynooth University, Maynooth, Co. Kildare, Ireland under path constraints through solving the open-loop optimal control problem on line. Generalised Model predictive control (GPC) was developed, based on a strong mathematical foundation, in parallel with the industry development of MPC (Clarke et al. 1987). Stability, robustness and feasibility were later investigated (Lee and Yu 1997; Mayne et al. 2000), giving a strong mathematical background to the MPC methodology (Lee 2011). Recent studies focused on decreasing the computational time needed to run MPC algorithms by adapting standard interior-point optimisation algorithms (Rao et al. 1998), or exploiting the structure of MPC problem to compute control laws off-line (Wang and Boyd 2010).

MPC has recently been introduced in the wave energy field with the objective of maximising the extracted energy from waves through controlling immersed wave energy converters (WECs), while satisfying technological constraints, such as stroke, control force or power limitations.

Several studies in the wave energy field adapted and optimised MPC algorithms specifically for WECs (Hals et al. 2011). An improvement of the state-space order used to model the WEC dynamics involved in the MPC algorithm was presented in Cretel et al. (2011), allowing an increase of the minimal time-step of the algorithm. Nonlinear MPC was implemented on a wave energy device in Richter et al. (2013), allowing a better description of the immersed system dynamics. Finally, convexity of the quadratic problems involved in the MPC algorithm applied to a WEC is studied in $\mathrm{Li}$ and Belmont (2014a), and its application to an array of WECs was then conducted in Li and Belmont (2014b).

An alternative approach to solve optimal control problems based on pseudospectral method has recently been developed and widely used in various applications (Elnagar et al. 1995; Gong et al. 2007; Ross and Fahroo 2004; Williams 2004a). Describing the state and control variables via a set of orthogonal basis functions, such as orthog- 
onal polynomials (Williams 2004b) or Fourier functions (Manita 2011; Nagurka and Yen 1990), pseudospectral optimal control allows a fast resolution of optimal trajectories for optimal control problems, while ensuring path constraints are respected, and subject to nonlinear system dynamics.

Pseudospectral optimal control has recently been introduced to the wave energy field, using Fourier basis functions for periodic optimal control (Bacelli and Ringwood 2015; Bacelli and Ringwood 2013). Applying a receding-horizon control, with an outer-loop based on pseudospectral optimal control, the optimal trajectories are determined and tracked in real-time in order to maximise the energy production, while respecting path constraints (Genest and Ringwood 2016).

These recent developments of advanced control strategies applied to wave energy converters have been able to provide real-time control algorithms that are able to handle realistic constraints and maximising the energy production of WECs. A comparison between a standard MPC and a pseudospectral optimal control algorithm, applied to wave energy devices, is presented in this paper. Their computational time and performances are presented for regular waves and irregular sea states under path constraints. This comparative study highlights the advantages and drawbacks of both methods and more importantly, brings additional suggestions for further research.

Section 2 introduces the standard optimal control problem and the practical constraints involved in the energy maximisation of a standard WEC described by linearised dynamical ordinary differential equations. Sections 3 and 4 present, respectively, the MPC and pseudospectral optimal control algorithms implemented for the comparison. Performance of the control strategies in regular and irregular waves is presented, respectively, in Sects. 6 and 7. Finally, a discussion, relating the advantages and drawbacks and the potential improvements of each method, is contained in Sect. 8.

\section{WEC model}

\subsection{Dynamical model}

In this study, a one-degree of freedom system is considered constrained to move in heave only. Referenced from its equilibrium position in an undisturbed wave field, immersed in an infinite-depth sea, the system is subject to fluid-structure interactions. The fluid is assumed to be inviscid; therefore, no viscous forces are considered in this study, and the motion of the WEC is assumed to be small enough to apply linearised potential flow theory for the determination of hydrodynamic forces. A generic $5 \mathrm{~m}$ diameter spherical shape is chosen for the WEC presented in Fig. 1.

Fluid-structure interaction forces acting on the system's hull can be determined as a sum of a radiation force $\mathbf{F}_{r}$, a

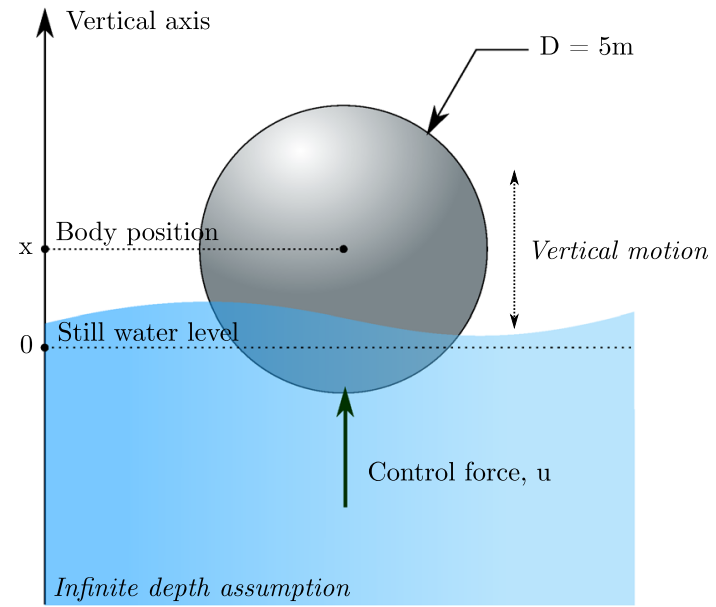

Fig. 1 Wave energy converter

hydrostatic force $\mathbf{F}_{h}$ and an excitation force $\mathbf{F}_{e}$. Hydrostatic forces for a floating device, i.e. buoyancy, is written as

$\mathbf{F}_{h}=\rho g V_{i} \mathbf{x}$,

where $\rho$ corresponds to the water density, $g$ is acceleration due to gravity, $\mathbf{x}$ is the vertical unitary vector and $V_{i}=V_{0}+$ $V(x)$ represents the immersed volume of the WEC, with $V_{0}$ the immersed volume at the equilibrium position and $V(x)$ the additional immersed volume depending on the position $x$ of the system. The weight of the wave energy device is $\mathbf{W}=-m g \mathbf{x}$, where $m=\rho g V_{0}$ is the total mass of the device. The sum $\mathbf{W}+\mathbf{F}_{h}=\rho g V(x) \mathbf{x}$ is linearised, assuming small body's displacements. The additional immersed volume is approximated by the following formula, $V(x) \approx-S x$.

The radiation force $\mathbf{F}_{r}$ is modelled using linearised potential flow theory, as a sum of a convolution product between the velocity of the device $v=\mathrm{d} x / \mathrm{d} t$ and a kernel function $K$, representing a memory term depending on past values of the velocity, and an added mass term proportional to the acceleration of the WEC's hull, as presented in (2):

$\mathbf{F}_{r}(t) \cdot \mathbf{x}=-\mu_{\infty} \frac{\mathrm{d} v}{\mathrm{~d} t}(t)-\int_{0}^{t} K(t-\tau) v(\tau) d \tau$

with $\mu_{\infty}$ corresponding to the infinite frequency added mass asymptote.

The excitation force $\mathbf{F}_{e}$ corresponds to the sum of the integration, on the WEC's hull, of the incident wave pressure field, and the diffraction force generated by the waves on the device. $\mathbf{F}_{e}$ can be determined, using linearised potential flow theory, via a standard boundary element method, from a convolution product between a kernel function and the free surface elevation of incoming waves.

The linearised equation of motion of the WEC, or Cummins' equation (Cummins 1962), projected on the $z$ axis, is written as 
$\left(m+\mu_{\infty}\right) \frac{\mathrm{d} v}{\mathrm{~d} t}+\int_{0}^{t} K(t-\tau) v(\tau) \mathrm{d} \tau+S_{h} x=F_{e}+u$,

where $u=F_{\text {PTO } \rightarrow \text { WEC }}$ is the control force applied on the system, $S_{h}=\rho g S$ corresponds to the hydrostatic stiffness and $F_{e}=\mathbf{F}_{e} \cdot \mathbf{x}$. The linear second-order integro-differential equation (3) describes the dynamical behaviour of the system subject to fluid-structure interactions and is used in both MPC and pseudospectral optimal control algorithms as a dynamic constraint.

\subsection{Path constraints}

Realistic approaches take into account physical limitations restraining the body's motion and its technological capacity, such as maximal actuator force and power. Path constraints avoid unrealistic trajectories and control forces, since optimal state and control variables, derived from the optimal complex-conjugate control (Falnes 2002), are often impracticable due to their considerable requirements. Moreover, practical aspects, such as the impact of actuator efficiency on the energy absorption (Genest et al. 2014), represent an additional justification for considering path constraints in the design of the control algorithm. Path constraints are written as

$\forall t \in \mathbb{R},\left(X_{\max }, V_{\max }, U_{\max }\right) \in \mathbb{R}^{+3},\left\{\begin{array}{l}|x(t)| \leq X_{\max } \\ |v(t)| \leq V_{\max } \\ |u(t)| \leq U_{\max },\end{array}\right.$

where $X_{\max }, V_{\max }, U_{\max }$ correspond to the position, velocity and control force practical limitations, respectively.

\subsection{Optimal control problem formulation}

The main objective of a WEC is to recover energy from the incoming wave field in which the device is immersed. This is done by applying a control force $u$ on the system's hull via a power takeoff (PTO). The role of the PTO is to convert the kinetic and potential energy of the device's motion into electric or mechanical energy. The power that fluctuates through the PTO, $P_{\mathrm{PTO} \rightarrow \text { WEC }}$, with all forces defined to be positive if applied along the $\mathbf{x}$ direction, is

$P_{\mathrm{PTO} \rightarrow \mathrm{WEC}}=F_{\mathrm{PTO} \rightarrow \mathrm{WEC}} V_{\mathrm{WEC} / R_{0}}=u v$

Thus, the absorbed energy $E$ of the WEC over a time period $T$ is

$E=\int_{0}^{T} P_{\mathrm{WEC} \rightarrow \mathrm{PTO}}(t) \mathrm{d} t=-\int_{0}^{T} u(t) v(t) \mathrm{d} t$

The objective of the control algorithm is to maximise the energy absorption while ensuring the respect of the dynamics of the system and the path constraints. The control objective is formulated, on the interval $I=[0, T]$, as follows:

$\max _{u, v \in C(I)} E(u, v)=-\int_{I} u(t) v(t) \mathrm{d} \tau$

subject to, for $t \in I$,

$$
\left\{\begin{array}{l}
\left(m+\mu_{\infty}\right) \frac{\mathrm{d} v}{\mathrm{~d} t}(t)+\int_{0}^{t} K(t-\tau) v(\tau) \mathrm{d} \tau+S_{h} x(t)=F_{e}(t)+u(t) \\
v(t)=\frac{\mathrm{d} x}{\mathrm{~d} t}(t) \\
|x(t)| \leq X_{\max } \\
|v(t)| \leq V_{\max } \\
|u(t)| \leq U_{\max }
\end{array}\right.
$$

MPC and RHPSC algorithms constitute different approaches in the resolution of the optimal control problem, maximising the energy absorption while ensuring path constraints and providing control and motion trajectories that follow the linearised dynamics of the system. They are individually described in Sects. 3 and 4, respectively.

\section{Model predictive control}

MPC has recently been applied to wave energy maximisation problems (Hals et al. 2011), allowing additional path constraints while providing a control law that maximises energy production. The convexity of the quadratic problem (QP), solved by a standard optimisation algorithm involved in MPC, has been studied in Li and Belmont (2014a) and the proposed method was applied to an entire array of WECs (Li and Belmont 2014b), illustrating the adaptability of the method.

Other parallel developments proposed a higher accuracy estimation of the dynamical equation by using a triangular hold, instead of the more traditional zero-order hold $(\mathrm{ZOH})$, while defining the cost function used for the MPC (Cretel et al. 2011). Nonlinear dynamical equations, providing more realistic WEC behaviour, were implemented in Richter et al. (2013), via nonlinear MPC (NMPC).

A real-time implementation of an MPC algorithm is presented in Fig. 2. It is decomposed into two parts, representing the trajectory generation and the simulation evaluation. To generate the trajectory, MPC solves the optimal control problem under constraints and provides, at each time-step, an optimal trajectory (future position, velocity and control force) within a fixed control horizon of duration $T$. The trajectory generation is updated at a predetermined fixed time step $\Delta t$. At each time-step, only the first output of the computed optimal force trajectory is applied to the device. The solution of the QP under constraints, therefore, becomes an input for the simulation evaluation. The evolution of the position and velocity of the device are determined in the real-time 
Fig. 2 MPC algorithm

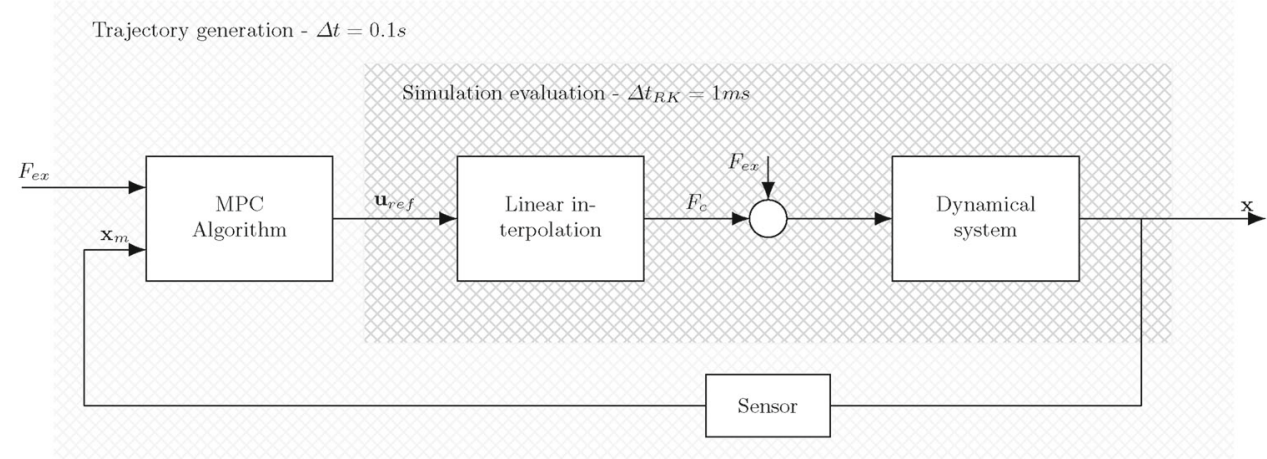

loop in the simulation evaluation, using a Runge-Kutta temporal discretization with a smaller time step $\Delta t_{\mathrm{RK}}$. In order to use the solution of the optimal control problem, the control force is linearly interpolated between two consecutive values provided by the MPC algorithm, allowing its implementation in the simulation evaluation.

\subsection{Continuous state-space model}

In order to use an MPC methodology, the dynamical model of the WEC is generally expressed as a state-space model, since the state and control variables are expressed in a discretetime formulation. Various ways can be employed to generate a state-space model from the Cummins' equation (3), and specifically to convert the convolution product in (2) involved in the radiation force into a finite-order parametric discretetime form (Kristiansen et al. 2005; Pérez and Fossen 2008).

In this study, two different methods are employed to generate a state-space model of the radiation convolution product. The first method realises a least-squares fitting of a transfer function to the radiation force in the frequency domain (Perez and Fossen 2009), while insuring the stability and passivity of the created state-space model. The second method is based on Prony's method (Duclos et al. 2001) and decomposes the radiation kernel function $K$ into a sum of complex exponential functions and uses this approximation to derive a continuous state-space model.

These two strategies provide the matrix $A_{r} \in \mathbb{R}^{n \times n}, B_{r} \in$ $\mathbb{R}^{n \times 1}$ and $C_{r} \in \mathbb{R}^{1 \times n}$, as

$$
\begin{aligned}
\dot{x}_{r}(t) & =A_{r} x_{r}(t)+B_{r} v(t) \\
\int_{0}^{t} K(t-\tau) v(\tau) \mathrm{d} \tau & \approx C_{r} x_{r}(t),
\end{aligned}
$$

where $x_{r} \in \mathbb{R}^{n \times 1}$ is a state-variable vector used to determine the radiation force. Its dimension depends on the approximation order, called $n$, in Prony's method (Duclos et al. 2001) or in the NTNU toolbox (Perez and Fossen 2009). Therefore, a continuous state-space model describing the complete Cummins' equation of (3) is defined in (12):

$$
\begin{aligned}
\dot{x}_{c} & =A_{c} x_{c}(t)+B_{c} F(t) \\
y_{c}(t) & =C_{c} x_{c}(t),
\end{aligned}
$$

where $x_{c}=\left[x, v, x_{r}^{\mathrm{T}}\right]^{\mathrm{T}} \in \mathbb{R}^{(n+2) \times 1}$ corresponds to the statevariable vector for a continuous time state-space model and the output vector $y_{c}(t)=[x(t), v(t)]^{\mathrm{T}} \in \mathbb{R}^{2 \times 1}$ provides the position and velocity of the WEC at the instant $t$. $F$ corresponds to the sum of the control force $u$ and the wave excitation force $F_{e}$, normalized by the total mass of the system $m_{T}=m+\mu_{\infty}$.

$A_{c}=\left[\begin{array}{lll}0 & 1 & 0 \\ -\frac{S_{h}}{m_{T}} & 0 & -C_{r} \\ 0 & \frac{B_{r}}{m_{T}} & A_{r}\end{array}\right] \in \mathbb{R}^{(n+2) \times(n+2)}$;

and,

$B_{c}=\left[\begin{array}{l}0 \\ 1 \\ 0\end{array}\right] \in \mathbb{R}^{(n+2) \times 1} ; \quad C_{c}=\left[\begin{array}{lll}1 & 0 & 0 \\ 0 & 1 & 0\end{array}\right] \in \mathbb{R}^{2 \times(n+2)}$

\subsection{State-space model discretization}

The chosen MPC algorithm is based on Cretel et al. (2011), since the continuous to discrete time transformation is realised using a first-order hold $(\mathrm{FOH})$, or triangular hold. The control force $u$ and the wave excitation force $F_{e}$ are then considered linear piecewise functions, instead of constant piecewise functions as with a standard $\mathrm{ZOH}$. The $\mathrm{FOH}$ generates a more precise model and allows larger simulation time-steps and, therefore, reduces the computational time of the MPC algorithm. The FOH used for the transformation from continuous to discrete time is shown in (15):

$$
\begin{aligned}
x_{d}[k+1]= & \Phi(\Delta t) x_{d}[k] \\
& +\int_{k \Delta t}^{(k+1) \Delta t} \Phi((k+1) \Delta t-\tau) B_{c}\left(u(\tau)+F_{e x}(\tau)\right) d \tau,
\end{aligned}
$$


with $\Phi(t)=e^{A_{c} t}$ and $x_{d}$ being the discrete-time quantities derived from sampling the variable $x_{c}$ at a $\Delta t$ time period. Using a FOH formulation for $u$ and $F_{e}$, we get

$$
\begin{aligned}
x_{d}[k+1]= & \Phi(\Delta t) x_{d}[k]+\Gamma\left(u_{d}[k]+F_{e, d}[k]\right) \\
& +\Lambda\left(\Delta u_{d}[k+1]+\Delta F_{e, d}[k+1]\right),
\end{aligned}
$$

where

$\Delta u_{d}[k+1]=u_{d}[k+1]-u_{d}[k]$

$\Delta F_{e, d}[k+1]=F_{e, d}[k+1]-F_{e, d}[k]$,

and, with $\Gamma=A_{c}^{-1}(\Phi(\Delta t)-I) B_{c}$ and $\Lambda=A_{c}^{-1}(\Gamma-$ $\left.\Delta t B_{t}\right) / \Delta t$, the discrete-time state-space model of the dynamical system becomes

$X[k+1]=A X[k]+B \Delta u_{d}[k+1]+F \Delta F_{e, d}[k+1]$

$Y[k+1]=C X[k]$

with $X=\left[x_{d}^{\mathrm{T}}, u_{d}, F_{e, d}\right]^{\mathrm{T}}, Y=\left[y_{d}^{\mathrm{T}}, u_{d}\right]^{\mathrm{T}}$,

$A=\left[\begin{array}{lll}\Phi(\Delta t) & \Gamma & \Gamma \\ 0 & 1 & 0 \\ 0 & 0 & 1\end{array}\right]$

and,

$B=\left[\begin{array}{l}\Lambda \\ 1 \\ 0\end{array}\right], \quad F=\left[\begin{array}{l}\Lambda \\ 0 \\ 1\end{array}\right], \quad C=\left[\begin{array}{llllll}1 & 0 & 0 & \ldots & 0 & 0 \\ 0 & 1 & 0 & \ldots & 0 & 0 \\ 0 & 0 & 0 & \ldots & 1 & 0\end{array}\right]$

Using the discrete time state-space model, one can generate, for the $N$ next time-steps, the prediction vector $\bar{Y} \in$ $\mathbb{R}^{3 N \times 1}$ as

$\bar{Y}=\left[\begin{array}{l}Y[k+1 \mid k] \\ \vdots \\ Y[k+N \mid k]\end{array}\right]$,

where $Y[k+i \mid k]$ corresponds to the prediction of the variable $Y$ at the $i$-th future time-step, knowing the current value of $Y[k]$. Based on such estimations of the state and control variables, one can derive the optimal control solution that will maximise the energy production while ensuring the respect of the path constraints on a given number of iterations. For further information about the quadratic problem formulation from the discrete state-space formulation, the reader may refer to the MPC study presented in (Cretel et al. 2011). In the present work, the exact same methodology is employed to implement the MPC algorithm.

\subsection{Cost function and optimisation}

The cost function $J$, involved in the absorbed energy maximisation, is quadratic and represents the actual energy absorption made by the WEC for a given control trajectory $u_{d}$, assuming the future values of the excitation force are known over the control horizon.

$J(\bar{Y})=\frac{1}{2} \bar{Y}^{\mathrm{T}} H \bar{Y}$,

where $H$ is a matrix determined from the discrete-time statespace model of the considered system, in our linear case involving only constant coefficients, and being the Hessian of the cost function $J$. As shown in Li and Belmont (2014a), the quadratic cost function $J$ is not always convex, leading to an increase of the computational time and potential multiple local solutions. The cost function can be convexified, without significantly penalising the power absorption, by adding diagonal terms to the matrix $H$ weighted by the absolute value of its smallest eigenvalue $\lambda_{\min }(H)(\mathrm{Li}$ and Belmont 2014a). The resulting convex cost function can then be written as follows:

$J(\bar{Y})=\frac{1}{2} \bar{Y}^{\mathrm{T}} H \bar{Y}+\left|\lambda_{\min }(H)\right| \bar{Y}^{\mathrm{T}} \bar{Y}$

In order to obtain the profile of the control force $u_{d} \in$ $\mathbb{R}^{N \times 1}$ over the $N$ next time-steps, the following constrained quadratic solved:

$$
\begin{gathered}
\bar{Y}^{*}=\max _{\bar{Y} \in \mathbb{R}^{(3 N \times 1)}}(J(\bar{Y})) \\
\text { s.t. }\left[\begin{array}{l}
\bar{Y} \\
-\bar{Y}
\end{array}\right] \leq I_{2 N} \otimes\left[\begin{array}{l}
X_{\text {max }} \\
V_{\text {max }} \\
U_{\text {max }}
\end{array}\right]
\end{gathered}
$$

where $\otimes$ represents the Kronecker product and $I_{2 N}=$ $[1, \ldots, 1]^{\mathrm{T}} \in \mathbb{R}^{2 N \times 1}$. One can notice that, in the MPC formulation, the ordinary differential equations describing the WEC dynamics are not considered as constraints, but are already implicitly expressed inside the cost function $J$. Standard algorithms, such as interior-point or active-set methods, can be employed to solve such quadratic problems under inequality constraints. The optimisation algorithm will give, at each time-step $\Delta t$, an optimal vector $\bar{Y}^{*}$. Only the first value of the control force derived from $\bar{Y}^{*}$ will be applied to the WEC. The procedure is then repeated to give a linear piecewise control force on-line.

\section{Receding horizon pseudospectral control}

Previous studies on pseudospectral control applied to wave energy devices were presented in Bacelli and Ringwood 
Fig. 3 RHPSC algorithm

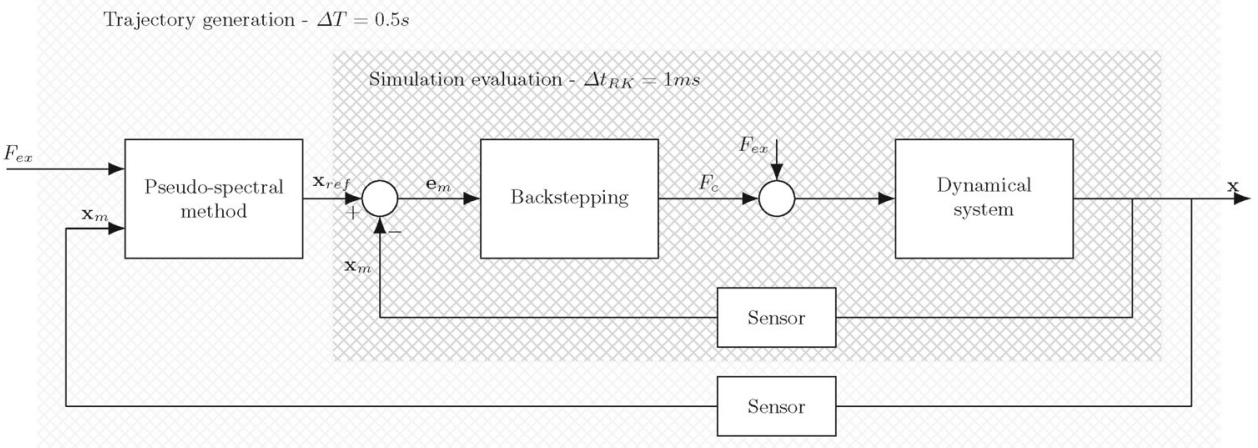

(2013, 2015) and use truncated Fourier series to approximate state and control variables. This method was presented for periodic signals over one or several periods, in regular or irregular waves, and shows promising results in maximising the energy production under path constraints within a short computational time. The Fourier basis used is particularly adapted for the approximation of periodic signals, but presents drawbacks when employed for non-periodic functions. Since the state and control variables are non-periodic, i.e. they do not have necessarily the same initial and final values over the control horizon, using truncated Fourier series to approximate state variables induces the well-known Gibbs phenomenon (Jerri 2013), since discontinuities will exist at the boundaries of the control horizon.

In order to circumvent the Gibbs phenomenon, the approximation of the state and control variables by their truncated Fourier series is replaced by their projections on a family of orthogonal polynomials, called Half-range Chebyshev Fourier (HRCF), defined in Huybrechs (2010). Following the development in Genest and Ringwood (2016), state and control variables are approximated in (28).

$x(t) \approx \sum_{i=0}^{N} a_{k} T_{k}^{h}\left(\cos \frac{\pi t}{2}\right)+\sum_{i=0}^{N-1} b_{k} U_{k}^{h}\left(\cos \frac{\pi t}{2}\right) \sin \frac{\pi t}{2}$,

where the coefficients $a_{k}$ and $b_{k}$ correspond to the orthogonal projections of the function $x$ on to the half-range Chebyshev Fourier basis

$a_{k}=\int_{-1}^{1} x(t) T_{k}^{h}\left(\cos \frac{\pi t}{2}\right) \mathrm{d} t$

and

$b_{k}=\int_{-1}^{1} x(t) U_{k}^{h}\left(\cos \frac{\pi t}{2}\right) \sin \frac{\pi t}{2} \mathrm{~d} t$

The continuous function $x$, approximated by the sum of its $2 N+1$ projections, is replaced in the optimal control problem, simplifying calculation of the optimal values of the $a_{k}$ and $b_{k}$ coefficients. The state and control variables are then represented by the vectors of their respective projections, e.g. in the case of the continuous $x$ function, $\hat{x}=\left[a_{0}, \ldots, a_{N}, b_{0}, \ldots, b_{N-1}\right]^{\mathrm{T}}$.

A receding horizon pseudospectral optimal control (RHPSC) was presented in Genest and Ringwood (2016), introducing a real-time control algorithm based on the pseudospectral method. The algorithm is used to control the wave energy device presented in Fig. 3. The optimal trajectories of $x, v$ and $u$ are determined via a pseudospectral method and tracked in real-time by a backstepping method (Krstic et al. 1995). In the same way as for the MPC algorithm, a Runge-Kutta temporal discretization, with a smaller time step $\Delta t_{R K}$ than the one used for the trajectory generation, is performed to obtain the real position and velocity of the device regardless of the model employed inside the control algorithm. This ensures a common simulation platform to allow a fair comparison of MPC and RHPSC performance.

\subsection{Dynamical equation}

In order to solve the optimal control problem via the pseudospectral method, the dynamical equation, in this case the Cummins' equation (3), needs to be expressed as a function of the vectors $\hat{x}, \hat{v}, \hat{u}$ and $\hat{F}_{e}$, corresponding to the orthogonal projections of, respectively, the position, velocity, control force and excitation force, on the half-range Chebyshev Fourier basis. In order to express the derivatives of variables in Cummins' equation (3), a differentiation matrix $D$ is necessary and defined in Orel and Perne (2012). Thus, the relation between the velocity and the position of the WEC, $v=d x / d t$, can be directly expressed with their projection vectors $\hat{v}$ and $\hat{x}$ in (31).

$\hat{v}=D \hat{x}$

where $D \in \mathbb{R}^{(2 N+1) \times(2 N+1)}$ is the differentiation matrix defined in Orel and Perne (2012).

The same methodology is employed to determine the convolution product arising in the expression of the radiation 
force. The projection vector of the convolution product $\hat{c}$, between the velocity and the radiation kernel function, is expressed as a product with a matrix $P$ and $\hat{v}$ in (32).

$\hat{c}=P \hat{v}$

The matrix $P$ contains the projections of the convolution between each basis function and the radiation kernel function $K$, for more details see (Genest and Ringwood 2016).

The Cummins' equation (3) is rewritten as a function of the projection vector of the state and control variables:

$$
\left(\left(m+\mu_{\infty}\right) D+P\right) \hat{v}+S_{h} \hat{x}=\hat{u}+\hat{F}_{e}
$$

\subsection{Cost function and optimisation}

The cost function $J$ representing the absorbed energy is determined by integrating the product of the velocity $v$ and the control force $u, J=-\int_{0}^{T} v u \mathrm{~d} t$ over the control horizon. Since all the basis functions are mutually orthogonal, the cost function is then simplified when replacing the state and control variables by their projections in the HRCF basis:

$J(Y)=-\frac{1}{2} Y^{\mathrm{T}} H Y$,

where $Y=\left[\hat{x}^{\mathrm{T}}, \hat{v}^{\mathrm{T}}, \hat{u}^{\mathrm{T}}\right]^{\mathrm{T}}$,

$H=\left[\begin{array}{lll}0 & 0 & 0 \\ 0 & 0 & I \\ 0 & I & 0\end{array}\right] \in \mathbb{R}^{3(2 N+1) \times 3(2 N+1)}$

and $I \in \mathbb{R}^{(2 N+1) \times(2 N+1)}$ representing the identity matrix.

The inequality constraints are also expressed, by replacing the state and control variables by their projections in the HRCF basis. Thus, the optimal control problem becomes a quadratic optimisation problem under equality and inequality constraints and can be solved using standard algorithms, such as interior-point or active-set methods. The equality constraints correspond to Eqs. (31) and (33) and the inequality constraints are collocated at $N_{c}$ Chebyshev points of the second kind (Genest and Ringwood 2016).

\section{Tuning of MPC and RHPSC algorithms}

By modelling the dynamical equation of the WEC using a state-space formulation, or by projection on a particular basis of orthogonal functions, both MPC and RHPSC formulations lead to a quadratic optimisation problem under constraints that can be solved using standard optimisation algorithms. An active-set method is employed for MPC and RHPSC, using the quadprog function from the optimisation toolbox of the MATLAB software.
The interior-point method proposed in the MATLAB optimisation toolbox was also employed to solve the resulting QPs derived from the RHPSC and MPC formulations. For both controls, under path constraints, the active-set method offers better performances in terms of computational time for MPC and performance for RHPSC. Despite a drop of energy absorption (around $10 \%$ ) for the RHPSC, while using the interior-point method under path constraints, its computational time remains at least 3 times smaller than the MPC one. No changes are observed in the unconstrained case. In contrast, MPC shows a significant increase of its computational time while using the interior-point method without path constraints. Thus, the active-set method was chosen to solve the QP involved in both controllers.

One interesting difference between the two formulations is the way the trajectory is computed during the optimisation process. The results shown for the MPC algorithm are generated with a simulation evaluation using the exact same model (state-space model) as the one employed to define the control MPC algorithm. In this way, the presented convergence curves do not depend on the quality of the state-space model approximation.

For the MPC algorithm, the state-space is repeatedly employed to derive estimations of future state variable values, leading to a matrix size that depends on the number of future steps taken into account over the control horizon. One should note that the accuracy of the MPC solution essentially depends on the time-step used for the simulation, keeping in mind that the excitation force is assumed to be a linear piecewise function, due to the continuous to discrete-time transformation. The MPC algorithm, presented here, is realised with two different methodologies to approximate the convolution product involved in the radiation force by a discrete state-space model. The first one, called here NTNU Toolbox, provides a 4th order discrete state-space model based on the algorithm presented in Perez and Fossen (2009). The second one, based on the Prony's method, returns a sum of four complex exponential functions representing the radiation kernel function $K$, as illustrated in Babarit and Clément (2006).

The order of each approximation model is chosen to strike a compromise between approximation accuracy and the stability of the approximation model. The influence of the approximation model order seems to be minimal, but a specific study needs to be carried out to answer this specific question that goes beyond the scope of the present paper. However, the approximation orders chosen (effectively 4 frequencies in both cases) are consistent with other reported experiences, e.g. Cretel et al. use a 5th order Pronys method (Cretel et al. 2011) and Hals et al. 4th order (Hals et al. 2011).

In contrast, RHPSC considers the dynamical equation as an equality constraint. The accuracy of the solution obtained through the pseudospectral optimal control obvi- 

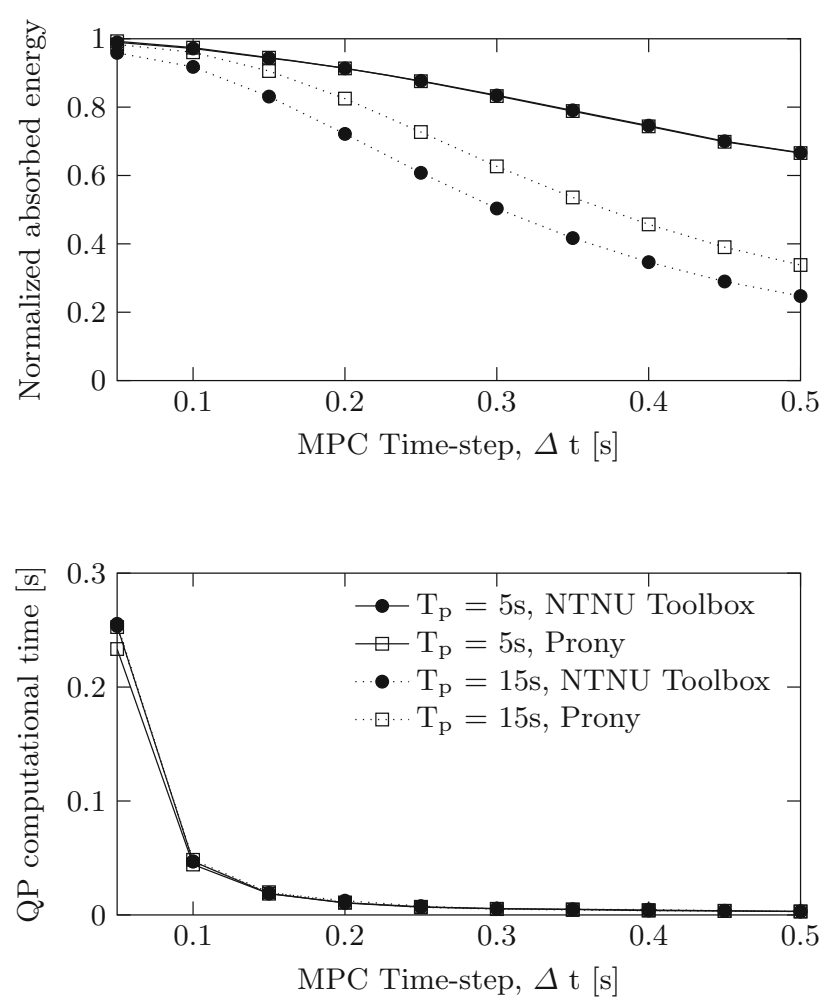

Fig. 4 Normalised absorbed power and QP computational time as a function of the time-step for the MPC simulation

ously depends on the number of basis functions employed in the approximation of the state and control variables. The more the basis functions involved in the optimization process, the better the trajectory definition accuracy. However, the accuracy of the dynamical model is fixed, since it is computed off-line via the calculation of the differentiation and convolution matrix, i.e. $D$ and $P$, respectively. Even if the precise calculation of $D$ and $P$ takes an significant amount of computational time, it has no effect on the on-line computation of the RHPSC quantities.

\subsection{MPC tuning}

Parameters involved in the computation of the MPC algorithm are the time-step $\Delta t \in \mathbb{R}^{+}$, used to compute the on-line control force, and the control window $T \in \mathbb{R}^{+}$, i.e. the time window over which the absorbed energy is maximised. In order to determine the optimal parameter values, several simulations were conducted in a regular wave field, without any path constraints, and compared with the optimal solution determined analytically from complex-conjugate theory (Falnes 2002).

Figure 4 shows the evolution of the normalised absorbed power and the computational time needed to solve the quadratic problem (QP) in equation (26), as a function of the MPC time-step, $\Delta t$. The convergence curves are presented for two types of regular waves, with a wave period of
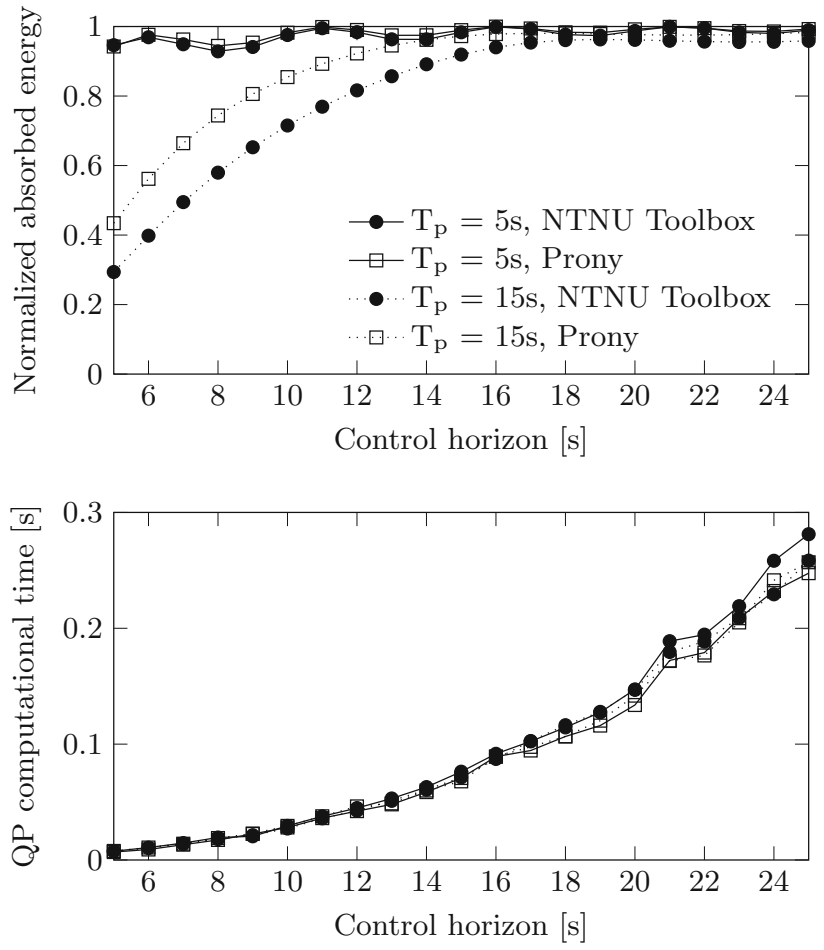

Fig. 5 Normalised absorbed power and QP computational time as a function of the control horizon for the MPC simulation

5 and $15 \mathrm{~s}$, corresponding, respectively, to the minimum and maximum values of the range of more probable incoming wave periods. The two methodologies used to approximate the convolution product of the radiation force for the MPC model, namely the NTNU Toolbox and Prony methods, are compared.

To make it clear to the reader, the present paper is not focused on a comparison of the state-space approximation methodologies, but simply exhibits the variabilities one can expect while using different state-space modelling methods. No conclusion on the relative quality of the presented methods can be drawn, since such a conclusion requires a complete comparison that is beyond the scope of this paper.

The absorbed energy is normalised by the theoretical maximum derived from the complex-conjugate theory (Falnes 2002). Both MPC implementations, with either a Prony parametrization or the NTNU Toolbox state-space approximation of the radiation force, give the same results in terms of computational time and energy absorption even if slight differences can be expected for large wave periods. The optimal value of the time-step is found to be around $\Delta t=0.1 \mathrm{~s}$, giving the best compromise between accuracy and computational time.

The change of the normalised absorbed power and computational time with the control horizon, or the time window under which the QP maximizes the energy absorption, is presented in Fig. 5. Similarly, the MPC with either Prony 
or NTNU-toolbox radiation force parametrization exhibits similar results. One can notice that, as expected, the computational time increases quadratically with an increase in the control horizon. Obviously, for a given time-step $\Delta t$, the longer the control horizon, the more the variables involved in the QP optimisation.

Some oscillations can be observed on the normalised energy absorption in Fig. 5, particularly for small wave periods. One probable explanation is the fact that the MPC algorithm, designed to maximise the absorbed energy over a fixed time window, is also trying to maximise the kinetic and potential energy absorption on the same control horizon. Thus, the position and velocity are brought back to zero by the QP optimisation algorithm at the end of the control horizon. This phenomenon might cause these oscillations, directly linked with the incoming wave period. A reasonable value for the control horizon duration is found to be around $15 \mathrm{~s}$, leading to an acceptable compromise between energy absorption and control horizon duration.

\subsection{RHPSC tuning}

The performance of the RHPSC algorithm mainly depends on two specific parameters: the number of basis functions involved in the approximation of the state and control variables $N \in \mathbb{N}$ and the duration of the control horizon $T \in \mathbb{R}^{+}$. The optimal values of the RHPSC parameters are determined to give the best compromise between accuracy and computational time, using the following considerations:

By way of comparison, the number of basis functions for the RHPSC case is generally smaller than that required for a similar level of approximation by the (effective) trapezoidal basis functions employed by MPC. The number of basis functions in the MPC case, however, is uniquely defined by the prediction horizon and the sampling period (Number of basis functions: $N_{b}=H_{p} / \Delta t$ ). In this sense, MPC could be considered as a class of spectral method with trapezoidal basis functions; for another example, see (Marzban and Razzaghi, 2010). The lower number of required basis functions for the RHPSC method suggests that the HRCF is more customised to the wave energy application than the trapezoidal function.

The change of the normalised absorbed energy and the QP computational effort for a different number of basis functions is presented in Fig. 6. The results are presented for regular incoming waves of 5 and $15 \mathrm{~s}$ periods. The small wave period case represents, for the RHPSC, the worst case of approximation, since the ratio between the period of the waves and the control horizon is small. Thus, more HRCF basis functions are needed to correctly approximate the state and control variables. One can see that after 21 basis functions, corresponding to $N=10$, the normalised absorbed energy has already converged, whereas the computational time needed to solve the QP is increasing. A reasonable choice for the
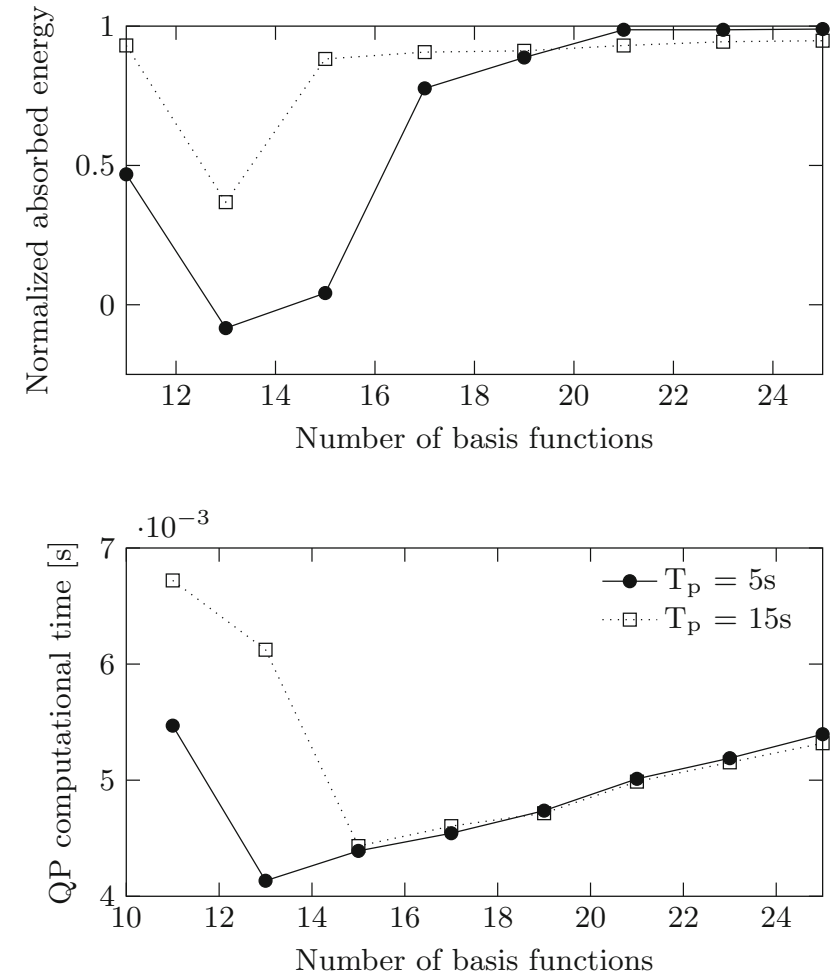

Fig. 6 Normalised absorbed power and QP computational time as a function of the number of HRCF basis functions in the RHPSC simulation

number $N$ is, therefore, found around 10 , leading to $21 \mathrm{HRCF}$ basis functions involved in the RHPSC algorithm.

Figure 7 presents the evolution of the normalised absorbed energy and the QP computation time for the RHPSC, plotted against the duration of the control horizon $T$. One can observe that, in contrast with the MPC algorithm, the computational time does not depend on the control horizon, since the number of basis functions are fixed, and consequently the size of the QP computational effort does not change as well. A reasonable choice for the control horizon will be a duration of 20 seconds.

Based on the selection of parameters for the MPC and RHPSC methods, as described in Sects. 5.1 and 5.2, respectively, comparisons between the presented methods are now performed with both regular and irregular sea states. For the remainder of the study, the chosen parameters and control windows are fixed as shown in Table 1.

\section{Relative performance in regular waves}

Based on the parameters defined in Table 1, the MPC and RHPSC algorithms were tested for regular waves with a wave period varying from 5 to $15 \mathrm{~s}$. The evolution of the absorbed energy and the computational time involved in the QP 

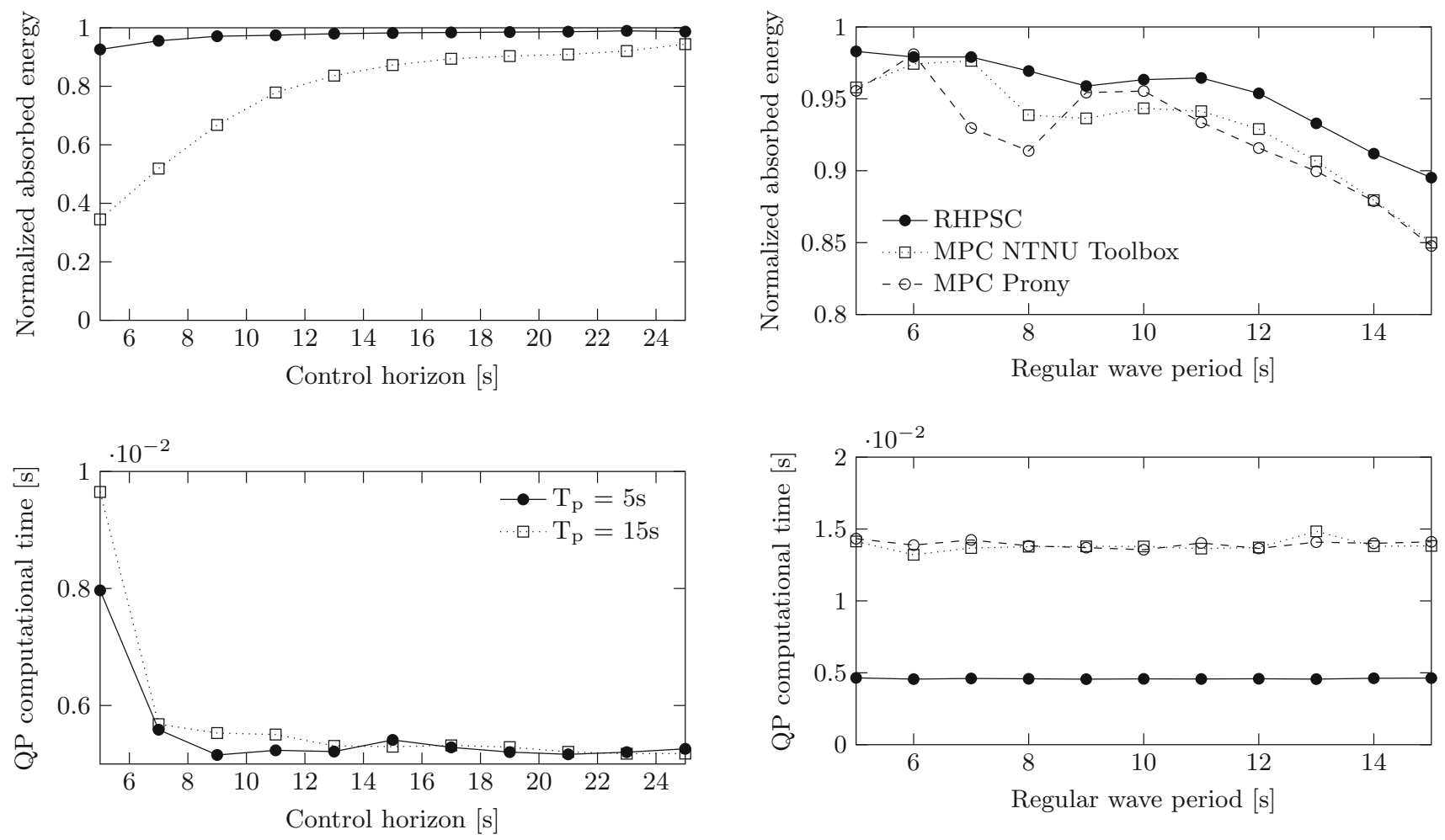

Fig. 7 Normalised absorbed power and QP computational time as a function of control horizon for the RHPSC method

Fig. 8 Normalised absorbed power and QP computational time for MPC and RHPSC algorithm for different regular wave periods

Table 1 Values of the MPC and RHPSC parameters

\begin{tabular}{lll}
\hline Algorithm & MPC & RHPSC \\
\hline Control horizon & $H_{p}=15 \mathrm{~s}$ & $H_{p}=20 \mathrm{~s}$ \\
Parameters & $\Delta t=0.1 \mathrm{~s}$ & $N=10$ \\
\hline
\end{tabular}

optimisation, as a function of the wave period, is presented in Fig. 8. The model used in the simulation evaluation loop is different from the dynamical model used to define the control algorithm, for both MPC and RHPSC. In the simulation evaluation, the convolution product involved in the determination of the radiation force is computed by a direct integration of the product between the past values of the real WEC velocity and the kernel function of radiation obtained from a boundary element method, to ensure an even comparison with maximum fidelity of the radiation force.

Both MPC implementations lead to comparable results; their energy absorption lies between 85 and $100 \%$ of the theoretical maximum. The computational time does not depend on the wave period of the incoming waves and is around $15 \mathrm{~ms}$. Since the time-step of the MPC algorithm is equal to $\Delta t=0.1 \mathrm{~s}$, the control can be run in real-time.

RHPSC presents approximately the same energy absorption and recovers between 90 and $100 \%$ of the theoretical maximum. The computational time involved in the QP opti- mization does not vary with the wave period of incoming regular waves and is found to be around $5 \mathrm{~ms}$. The RHPSC presents relatively fast convergence property and reaches the same rate of energy absorption as a standard MPC algorithm, with a computational time around three time smaller.

The energy absorption performance of both control algorithms under path constraints is presented in Fig. 9. For these simulations the position of the device is limited to $\pm 0.1 \mathrm{~m}$ and its velocity to $\pm 0.1 \mathrm{~m} / \mathrm{s}$. The same control tuning defined in Table 1 is employed and the waves are regular, with periods between 5 and $15 \mathrm{~s}$.

The impact of the constraints on the energy absorption is similar for both the MPC and the RHPSC. For small wave periods, around $5 \mathrm{~s}$, the motion of the device does not exceed the constraints, and thus the constraints do not impact the energy absorption, found to be around $95 \%$ of the theoretical maximum. When the wave period increases, for a fixed wave amplitude, the excitation force increases as well. Yet, the natural motion of the device purports to exceed the position and velocity limitations, and the impact on the energy absorption becomes more significant as the wave period increases. The normalised energy absorption drops from $95 \%$ for a $5 \mathrm{~s}$ wave period to $15 \%$ for a $15 \mathrm{~s}$ wave period.

However, the focus of the study is not to present a qualitative estimation of the amount of energy loss due to path constraints, but rather to illustrate the ability of both control 

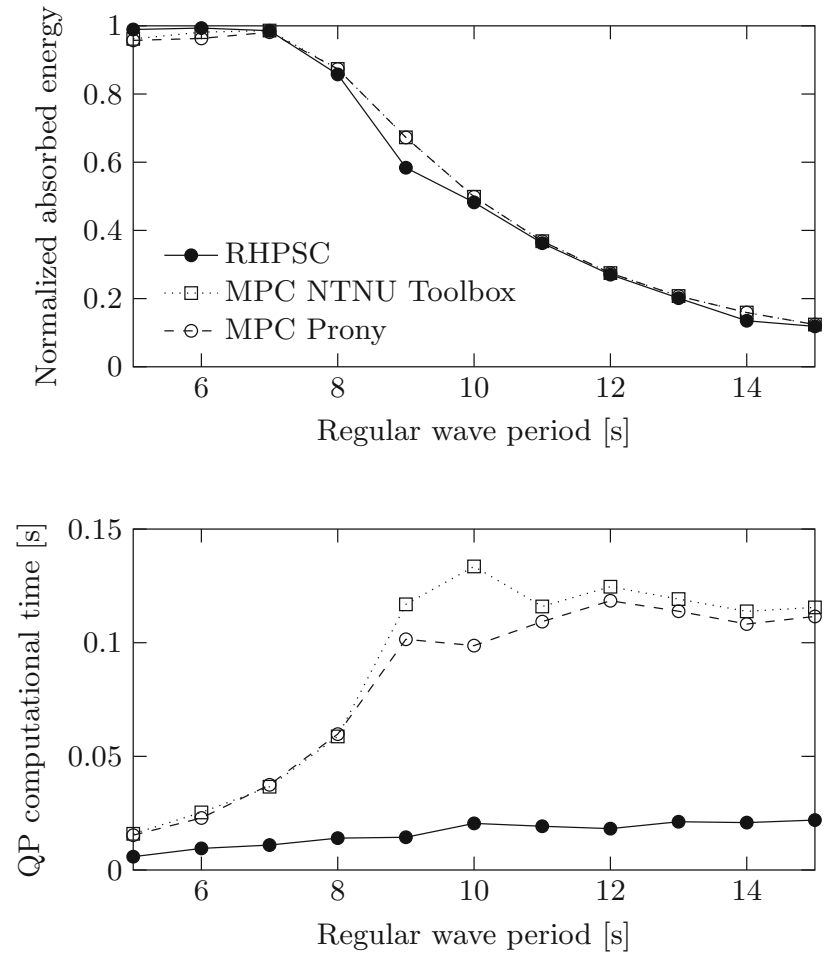

Fig. 9 Normalised absorbed power and QP computation time for MPC and RHPSC algorithms for different regular wave periods under position and velocity path constraints

algorithms to handle practical limitations, while maximizing the energy production. The impact of path constraints on the computational time to solve one particular QP with inequality constraints is shown in Fig. 9. Obviously, the presence of constraints tends to increase the computational time, since additional iterations will be needed in the active-set algorithm to maximize the energy while respecting inequality constraints. The MPC computational time seems to be more sensitive to inequality constraints than the RHPSC, since its computational time varies from $15 \mathrm{~ms}$ for a $5 \mathrm{~s}$ regular wave period to $0.1 \mathrm{~s}$ for regular waves with a period greater than $9 \mathrm{~s}$, which puts it on the limit of real-time implementation (with a MATLAB implementation). For the RHPSC, the computational time stays between 5 and $25 \mathrm{~ms}$. The difference in sensitivity under constraints between MPC and RHPSC may come from the fact that constraints on the state or control variables have a global impact on the trajectory for the pseudospectral method, since each basis function is defined over the entire control horizon, while the basis functions for MPC are defined locally.

\section{Relative performance in irregular waves}

The performance of the MPC and RHPSC are evaluated in irregular waves in order to simulate more realistic sea states. Results are presented for irregular waves generated from a Pierson-Moskowitz spectrum discretized in frequency between 0.02 and $0.5 \mathrm{~Hz}$, corresponding (respectively) to $50 \mathrm{~s}$ and $2 s$ periods, with a frequency step of $d f=5 \cdot 10^{-3} \mathrm{~Hz}$. Each spectral component is phase shifted randomly between 0 and $2 \pi$, and the absorbed energy is computed over a $200 \mathrm{~s}$ time window. The results are presented in Fig. 10, for irregular sea states with peak periods varying between $T_{p}=5 \mathrm{~s}$ to $T_{p}=15 \mathrm{~s}$. One can see that the relative performance is similar to the regular wave case, since the absorbed energy and the computational time do. One can notice a slight decrease in the energy absorption for large peak periods, around $T_{p}=15 \mathrm{~s}$. This is due to the fact that the MPC and RHPSC tunings are based on performance determined under regular wave constraints in a $[5 \mathrm{~s}, 15 \mathrm{~s}]$ period range.

To illustrate the ability of the MPC of RHPSC algorithms to maximize the energy production under unconstrained conditions, time series of the position, velocity, control force and absorbed energy are presented in Fig. 11, for an irregular sea state with a peak period of $10 \mathrm{~s}$. Both MPC and RHPSC trajectories are compared to the theoretical optimal one and present a relatively acceptable agreement. Oscillations can be noticed, in particular for the RHPSC, at the beginning of the simulation. The control algorithms starts to drive the WEC with an initial condition of zero, i.e. position and velocity null at the origin of time, while the theoretical optimal
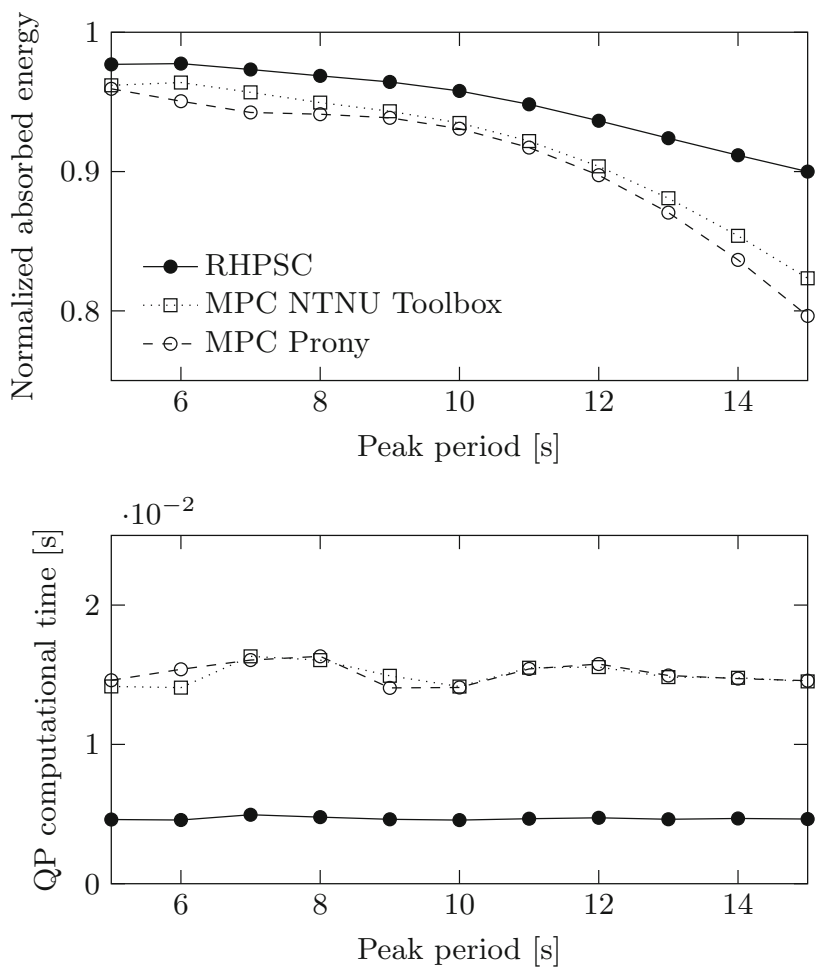

Fig. 10 Normalised absorbed power and QP computational time for MPC and RHPSC algorithm for different irregular sea states 
Fig. 11 Normalised absorbed power and QP computational time for MPC and RHPSC algorithms for different irregular sea states
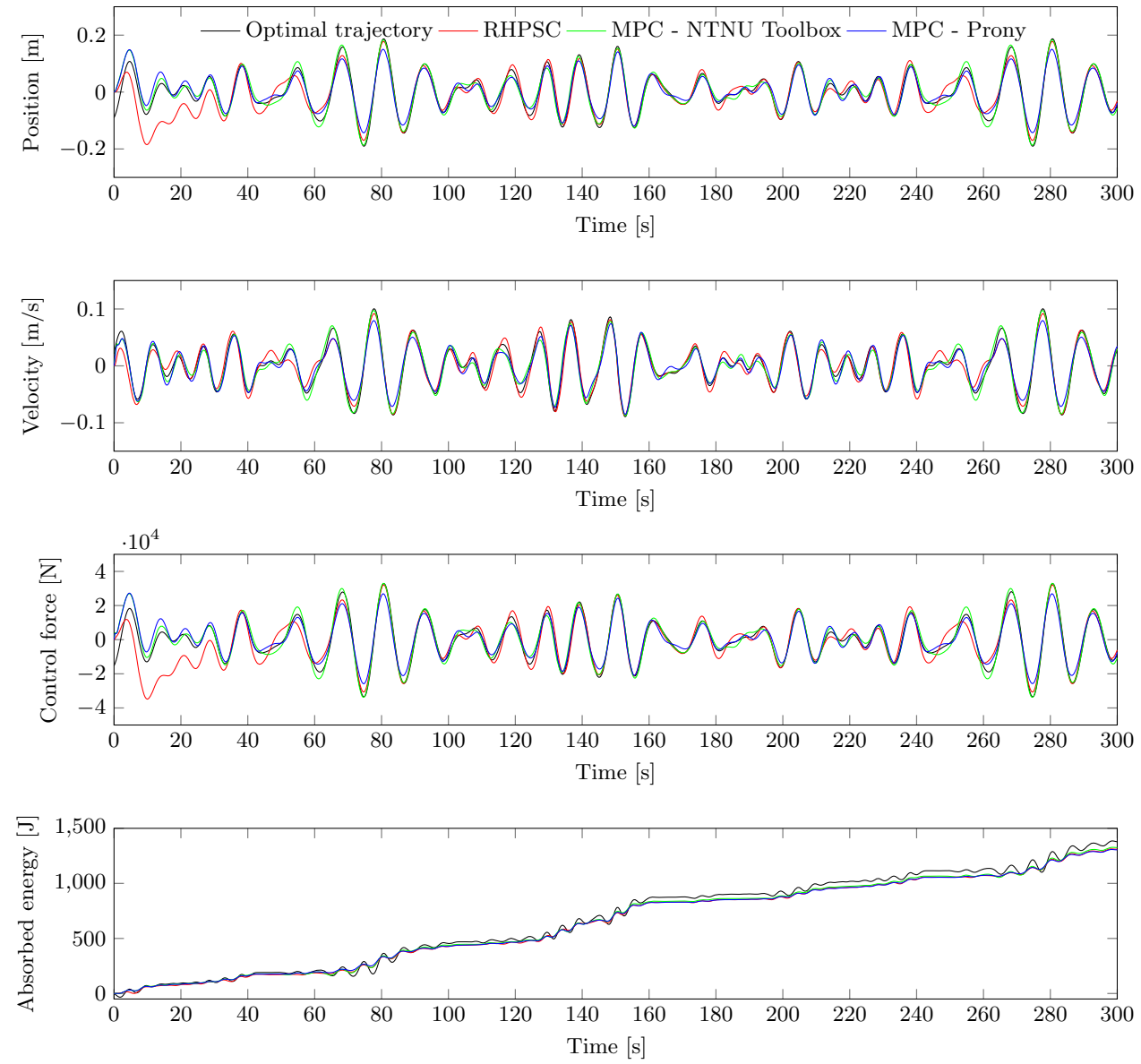

trajectory begins with non-null initial conditions. This difference induces a transient state necessary for the RHPSC control algorithm to reach the optimal trajectory from null initial conditions.

MPC and RHPSC are particularly interesting and practical control algorithms since they can handle constraints, or technological limitations, while maximizing the energy production. To illustrate the ability of the MPC and RHPSC algorithms under constrained conditions, the time series of the control and state variables, and their technological limitations, are show in Fig. 12 for an irregular sea state with a peak period of $10 \mathrm{~s}$.

\section{Discussion}

Based on the results presented in Sects. 6 and 7 for regular and irregular waves, some insights and further improvements can be deduced from these comparisons.

The MPC algorithm seems to present a sensitivity to the way the state-space model is designed. Different radiation force model were compared to achieve the most accurate models possible from the NTNU Toolbox and the Prony's method. This issue might not be noticed if the model used to evaluated the MPC algorithm is identical to that used to design the control. For this reason, the time-domain simulation was carried out with an exact calculation of the radiation force, instead of using the state-space employed in the MPC algorithm. The approximation of the radiation force by a state-space model is not unique and leads to a variety of techniques that might generate different performance and need to be assessed. On the other hand, RHPSC give a unique procedure to estimate the expression of the dynamical equations in terms of the projections of the state and control variables into the half-range Chebyshev Fourier basis. However, both MPC and RHPSC can be affected by inherent errors arising in the estimation of the radiation kernel function $K$. The sensitivity of both algorithms still needs to be investigated, since a recent experimental study (Kracht 2013) shows poor performance of MPC compared to a much simpler control algorithm (Fusco and Ringwood 2013). It is likely that the radiation force approximation, in addition to any other sensitivity to modelling error, played a significant part in this performance degradation.

Both MPC and RHPSC are suitable for real-time application, even if the RHPSC is around three times faster in generating an optimal trajectory. Even a MATLAB implementation gives an execution time of circa $0.3 \mathrm{~s}$ which, when 
Fig. 12 Normalised absorbed power and QP computational time for MPC and RHPSC algorithms for different irregular sea states under path constraints
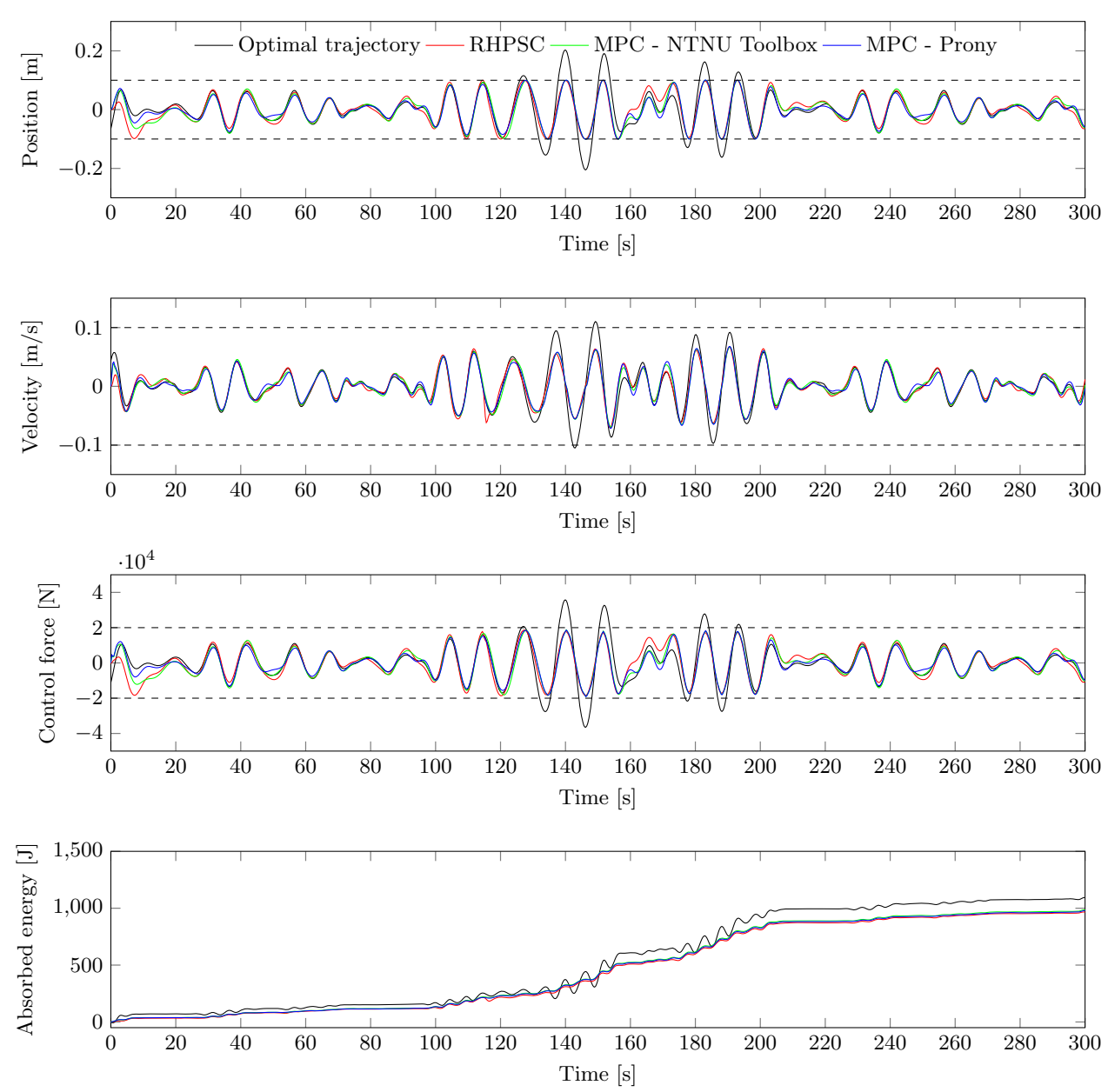

compared with the relatively slow dynamics of ocean WECs, would result in real-time feasibility for a $\mathrm{C}$ implementation. Furthermore, the possibility for computational optimisation of both MPC (Wang and Boyd 2010) and RHPSC for the wave energy application, with particular emphasis on the constrained optimisation problem, can also be investigated.

\section{Conclusion}

Both MPC and RHPSC algorithms show a level of performance approaching that of the theoretical optimum determined from the complex-conjugate optimal, for the unconstrained case. Across both unconstrained and constrained scenarios, the performance of RHPSC and MPC is comparable, when each is tuned optimally. However, the relative computational burden for MPC is significantly greater than for RHPSC, usually by a factor of 3 . However, both MPC and RHPSC exhibit computational properties that allow their real-time implementation in a WEC application, at least for linear hydrodynamic models, given the relatively slow dynamics of WECs and that fact that significant speed-up will be achieved in an embedded software realisation, com- pared to the interpreted Matlab implementations compared here. On the computational side, RHPSC methods have been shown to have useful properties in the application to complex nonlinear problems (Bedrossian et al. 2009), which could prove advantageous in more realistic WEC control. Though beyond the scope of this paper, the comparative performance of RHPSC and MPC utilising nonlinear WEC models would be an important next step. In this case, the control methods would likely be presented with a more difficult optimisation problem, as is the case for conventional MPC-like tracking problems (Tenny et al. 2004). Indeed, the determination of adequate hydrodynamic models is, of itself, a nontrivial task (Penalba Retes et al. 2015) though some progress has been made in robustifying WEC controllers to nonlinear effects (Fusco and Ringwood 2014).

MPC appears to have a sensitivity to the manner in which a finite-order parametrization of the radiation force is achieved, and significant effort must be expended in obtaining an acceptable approximation to give reasonable control results. While RHPSC also involves a projection of the radiation damping characteristics onto a basis function set, this process appears to be simpler and provides better fidelity than the state-space or Prony approximation required with MPC. In 
any case, the issue of the robustness of highly tuned numerical optimal WEC controllers needs further study, since additional modelling errors will arise due to unmodelled dynamics, nonlinearity, etc. This robustness issue is especially pertinent, since WEC controllers (which ostensibly operate in open loop) do not have the attractive sensitivity properties that traditional feedback controllers enjoy.

While robust control is a relatively mature field for traditional tracking problems, including pseudo-spectral control (Azhmyakov et al. 2015), robust control design for WECs has received little attention [apart from, for example, Fusco and Ringwood (2014)]. Given the relatively good performance of a simple model-based controller against its MPC counterpart under real modelling error conditions (Kracht 2013), the flexibility in the selection of the basis function set of pseudospectral methods may offer the control designer some useful parametric freedom in directly addressing the robust control problem for WECs.

Acknowledgments This paper is based upon work supported by Science Foundation Ireland under Grant No. 13/IA/1886.

\section{References}

Azhmyakov V, Shamsi M, Trujillo LAG, Rojas JHC (2015) A robust pseudo-spectral method for a class of optimal control problems. IFAC-PapersOnLine 48(14):253-258

Babarit A, Clément A (2006) Optimal latching control of a wave energy device in regular and irregular waves. Appl Ocean Res 28(2):77-91

Bacelli G, Ringwood J (2013) Constrained control of arrays of wave energy devices. Int J Mar Energy 3(4):e53-e69

Bacelli G, Ringwood JV (2015) Numerical optimal control of wave energy converters. IEEE Trans Sustain Energy 6(2):294-302

Bedrossian NS, Bhatt S, Kang W, Ross IM (2009) Zero-propellant maneuver guidance. Control Syst IEEE 29(5):53-73

Clarke D, Mohtadi C, Tuffs P (1987) Generalized predictive control part i. the basic algorithm. Automatica 23(2):137-148

Cretel JA, Lightbody G, Thomas GP, Lewis AW (2011) Maximisation of energy capture by a wave-energy point absorber using model predictive control. In: Proceedings of the 18th IFAC World Congress. Milano, Italy, Aug, pp 3714-3721

Cummins W (1962) The impulse response function and ship motions. Tech. rep., DTIC Document

Cutler CR, Ramaker BL (1980) Dynamic matrix control—a computer control algorithm. In: Joint automatic control conference, 17, p 72

Duclos G, Clément AH, Chatry G (2001) Absorption of outgoing waves in a numerical wave tank using a self-adaptive boundary condition. Int J Offshore Polar Eng 11(03):168-175

Elnagar G, Kazemi MA, Razzaghi M (1995) The pseudospectral legendre method for discretizing optimal control problems. IEEE Trans Autom Control 40(10):1793-1796

Falnes J (2002) Ocean waves and oscillating systems: linear interactions including wave-energy extraction. Cambridge University Press, Cambridge

Fusco F, Ringwood JV (2013) A simple and effective real-time controller for wave energy converters. IEEE Trans Sustain Energy 4(1):21-30

Fusco F, Ringwood JV (2014) Hierarchical robust control of oscillating wave energy converters with uncertain dynamics. IEEE Trans Sustain Energy 5(3):958-966
Genest R, Ringwood JV (2016) Receding horizon pseudospectral control for energy maximisation with application to wave energy devices. IEEE Trans Control Syst Technol PP(99):1-10. doi:10. 1109/TCST.2016.2554524

Genest R, Bonnefoy F, Clément AH, Babarit A (2014) Effect of nonideal power take-off on the energy absorption of a reactively controlled one degree of freedom wave energy converter. Appl Ocean Res 48:236-243

Gong Q, Kang W, Bedrossian NS, Fahroo F, Sekhavat P, Bollino K (2007) Pseudospectral optimal control for military and industrial applications. In: Decision and Control, 2007 46th IEEE Conference on, IEEE, pp 4128-4142

Hals J, Falnes J, Moan T (2011) Constrained optimal control of a heaving buoy wave-energy converter. J Offshore Mech Arct Eng 133(1):011401

Huybrechs D (2010) On the Fourier extension of nonperiodic functions. SIAM J Numer Anal 47(6):4326-4355

Jerri AJ (2013) The Gibbs phenomenon in Fourier analysis, splines and wavelet approximations, vol 446. Springer Science and Business Media, New York, USA

Kracht P (2013) Wave prediction and its implementation on control systems of wave-energy converters. Fraunhofer IWES, EU MaRINet Infrastructure Access Report, Tech Rep MARINET-TA1-Adaptive WEC control

Kristiansen E, Hjulstad Å, Egeland O (2005) State-space representation of radiation forces in time-domain vessel models. Ocean Eng 32(17):2195-2216

Krstic M, Kokotovic PV, Kanellakopoulos I (1995) Nonlinear and adaptive control design. Wiley, John Wiley \& Sons, Hoboken (New Jersey) USA

Lee J, Yu Z (1997) Worst-case formulations of model predictive control for systems with bounded parameters. Automatica 33(5):763-781

Lee JH (2011) Model predictive control: Review of the three decades of development. Int J Control Autom Syst 9(3):415-424

Li G, Belmont MR (2014a) Model predictive control of sea wave energy converters-part i: a convex approach for the case of a single device. Renew Energy 69:453-463

Li G, Belmont MR (2014b) Model predictive control of sea wave energy converters-part ii: The case of an array of devices. Renew Energy 68:540-549

Manita L (2011) Optimal control problems for wave equations. J Math Sci 177(2):257-269

Marzban H, Razzaghi M (2010) Rationalized haar approach for nonlinear constrained optimal control problems. Appl Math Model 34(1):174-183

Mayne DQ, Rawlings JB, Rao CV, Scokaert PO (2000) Constrained model predictive control: stability and optimality. Automatica 36(6):789-814

Nagurka M, Yen V (1990) Fourier-based optimal control of nonlinear dynamic systems. J Dyn Syst Meas Control 112(1):17-26

Orel B, Perne A (2012) Computations with half-range chebyshev polynomials. J Comput Appl Math 236(7):1753-1765

Penalba Retes M, Giorgi G, Ringwood J (2015) A review of non-linear approaches for wave energy converter modelling. In: Proceedings of the 11th European Wave and Tidal Energy Conference, European Wave and Tidal Energy Conference 2015

Pérez T, Fossen TI (2008) Time-vs. frequency-domain identification of parametric radiation force models for marine structures at zero speed. Model Identif Control 29(1):1-19

Perez T, Fossen TI (2009) A MATLAB toolbox for parametric identification of radiation-force models of ships and offshore structures Model Identif Control 30(1):1

Rao CV, Wright SJ, Rawlings JB (1998) Application of interiorpoint methods to model predictive control. J Optim Theory Appl 99(3):723-757 
Richalet J, Rault A, Testud J, Papon J (1978) Model predictive heuristic control: applications to industrial processes. Automatica 14(5):413-428

Richter M, Magaña ME, Sawodny O, Brekken TK (2013) Nonlinear model predictive control of a point absorber wave energy converter. IEEE Trans Sustain Energy 4(1):118-126

Ross IM, Fahroo F (2004) Pseudospectral methods for optimal motion planning of differentially flat systems. IEEE Trans Autom Control 49(8):1410-1413

Tenny MJ, Wright SJ, Rawlings JB (2004) Nonlinear model predictive control via feasibility-perturbed sequential quadratic programming. Comput Optim Appl 28(1):87-121
Wang Y, Boyd S (2010) Fast model predictive control using online optimization. IEEE Trans Control Syst Technol 18(2):267-278

Williams P (2004a) Application of pseudospectral methods for receding horizon control. J Guid Control Dyn 27(2):310-314

Williams P (2004b) Jacobi pseudospectral method for solving optimal control problems. J Guid Control Dyn 27(2):293-297 